\title{
Video Article \\ Stab Wound Injury of the Zebrafish Adult Telencephalon: A Method to Investigate Vertebrate Brain Neurogenesis and Regeneration
}

\author{
Rebecca Schmidt ${ }^{1}$, Tanja Beil ${ }^{1}$, Uwe Strähle ${ }^{1}$, Sepand Rastegar ${ }^{1}$ \\ ${ }^{1}$ Institute of Toxicology and Genetics, Karlsruhe Institute of Technology \\ Correspondence to: Sepand Rastegar at sepand.rastegar@kit.edu \\ URL: https://www.jove.com/video/51753 \\ DOI: doi:10.3791/51753
}

Keywords: Neuroscience, Issue 90, zebrafish, adult neurogenesis, telencephalon regeneration, stab wound, central nervous system, adult neural stem cell

\section{Date Published: 8/4/2014}

Citation: Schmidt, R., Beil, T., Strähle, U., Rastegar, S. Stab Wound Injury of the Zebrafish Adult Telencephalon: A Method to Investigate Vertebrate Brain Neurogenesis and Regeneration. J. Vis. Exp. (90), e51753, doi:10.3791/51753 (2014).

\section{Abstract}

Adult zebrafish have an amazing capacity to regenerate their central nervous system after injury. To investigate the cellular response and the molecular mechanisms involved in zebrafish adult central nervous system (CNS) regeneration and repair, we developed a zebrafish model of adult telencephalic injury.

In this approach, we manually generate an injury by pushing an insulin syringe needle into the zebrafish adult telencephalon. At different post injury days, fish are sacrificed, their brains are dissected out and stained by immunohistochemistry and/or in situ hybridization (ISH) with appropriate markers to observe cell proliferation, gliogenesis, and neurogenesis. The contralateral unlesioned hemisphere serves as an internal control. This method combined for example with RNA deep sequencing can help to screen for new genes with a role in zebrafish adult telencephalon neurogenesis, regeneration, and repair.

\section{Video Link}

The video component of this article can be found at https://www.jove.com/video/51753/

\section{Introduction}

Mammals have a very limited capacity to generate new neurons during adult life, and adult neurogenesis in the brain is mostly restricted to two telencephalic regions situated at the subventricular zone (SVZ) of the lateral ventricles, and the subgranular zone (SGZ) of the dentate gyrus in the hippocampus ${ }^{1}$. Mammals also cannot repair damage of the brain caused by neurodegenerative diseases, stroke, or injuries, efficiently. Lost neurons are not replaced and instead proliferation of different types of glial cells, including astrocytes, microglia, and oligodendrocytes, is observed. As a consequence of this proliferative process called reactive gliosis, the injured brain tissue is sealed off by the formation of a glial scar, which inhibits neuronal and axonal regeneration ${ }^{2-4}$.

In contrast to mammals, teleost fish like the zebrafish form new neurons abundantly in adult stages and have a high regenerative and proliferative potential to repair lesions of the central nervous system ${ }^{2,5-7}$. The zebrafish adult brain contains 16 distinct neural stem cell niches that generate a large number of new neurons during the entire life ${ }^{8-11}$. Neurons born in these specific proliferation zones of the adult zebrafish brain migrate to their target areas, where they will mature and integrate into the existing neuronal networks ${ }^{8,10,12-15}$.

Among the progenitor zones identified in adult zebrafish, the telencephalic ventricular zone is one of the most studied neuronal stem cell niches. Cells in this progenitor niche can be classified into three distinct types regarding their morphology, division rate and expression of different glial or neuronal markers. Type I and type II cells are radial glial like cells which have long processes. They express stem cells markers including GFAP, nestin, and S100ß. Type I cells do not proliferate while type II cells proliferate slowly, as evidenced by their expression of proliferation markers such as proliferating cell nuclear antigen (PCNA) and the incorporation of desoxythymidine analogues. The type III cells are fast dividing cells that are committed to become neural progenitors and express neural marker genes, such as PSA-NCAM ${ }^{5,16}$.

Although the regenerative capacities of teleost fish are well documented, only few publications have described and investigated in detail the cellular and molecular mechanisms involved in neuronal regeneration in the adult telencephalon in response to injury ${ }^{15,17-22}$. To decipher the mechanisms implicated in zebrafish adult central nervous system regeneration and repair and to understand the similarities and the differences between constitutive and regenerative neurogenesis, we developed a zebrafish model of adult telencephalic injury. Here, we will describe visually how to generate an injury in one of the telencephalic hemispheres with the help of a syringe needle, while keeping the contralateral unlesioned side as an internal control. We will also show how to monitor changes upon injury in cell proliferation and gene expression by immunohistochemistry and in situ hybridization assays. 


\section{Protocol}

Zebrafish were maintained in the fish facility of the Institute of Toxicology and Genetics (ITG) at Karlsruhe Institute of Technology (KIT).

Experiments on animals were performed in accordance with the German animal protection standards and were approved by the Government of Baden-Württemberg, Regierungspräsidium Karlsruhe, Germany (Aktenzeichen 35-9185.81/G-272/12 'Adulte Neurogenese').

\section{Generating a Stab Wound in the Telencephalon}

1. Anesthesia

1. To prepare a stock solution of tricaine (3-amino benzoic acid ethyl ester, also called ethyl 3-aminobenzoate) use $400 \mathrm{mg}$ of tricaine powder and dissolve it in $97.9 \mathrm{ml}$ water and $2.1 \mathrm{ml} 1 \mathrm{M} \mathrm{Tris} / \mathrm{HCl}$ buffer $(\mathrm{pH} 9)$. Adjust the $\mathrm{pH}$ to 7 . Make $5 \mathrm{ml}$ aliquots and store them at $-20{ }^{\circ} \mathrm{C}$ until usage.

2. Transfer an appropriate number of 0.5-1 year old zebrafish from their tanks into plastic mouse cages. Note: The skull of an adult zebrafish at about 12 months of age is still relatively soft and can be perforated easily with a needle (see section 1.2.3 and Figure 1A).

3. To anesthetize the adult zebrafish, combine $5 \mathrm{ml}$ tricaine (stock solution) in a plastic crossing cage with $\sim 100 \mathrm{ml}$ of clean fish tank water (final concentration of tricaine $0.02 \%(w / v)$ tricaine).

4. Incubate the fish in the anesthetics until they do not move anymore (45-60 sec).

2. Telencephalon Injury

1. Place individual anesthetized zebrafish into a slit in a block of tricaine-soaked foam.

2. Under a dissecting microscope with light from the top gently hold the fish with one hand and orient it in a way that allows access to the head from the top.

3. With the other hand push a $30 \mathrm{G}$ syringe (insulin syringe with integrated needle) vertically through the skull into the medial region of one telencephalic hemisphere (Figures 1A-1B). The hemispheres of the telencephalon are visible through the skull. Make sure that the introduced lesion is not deeper than $2 \mathrm{~mm}$ (Figure 1C). Note: It is highly recommended to introduce the lesion always into the same hemisphere to ease the identification of the site of lesion when dissecting the brain out of the skull.

4. After introducing the telencephalic injury, place the fish into fresh fish water. Keep up to 10 fish in a $2 \mathrm{~L}$ mouse cage filled with fish water for recovery and connect the mouse cage to a water flow system. Note: The survival rate is usually $\sim 97 \%$ if fish are healthy and no other experiments were performed with the fish before. Addition of antibiotics to the fish water is not necessary. After recovery, fish behave normally: they swim, feed, and mate.

\section{Analyzing the Effect of Telencephalic Injury}

1. Brain Dissection and Fixation

1. Re-anaesthetize the recovered fish at different time points after lesion (typically, $1,3,5$, and 14 days post lesion (dpl)) with $0.02 \%(w / v)$ tricaine as above and euthanize them by putting them into ice water for $5 \mathrm{~min}$.

2. Place the fish on a paper tissue soaked in phosphate-buffered saline (PBS) and separate the head from the body by cutting behind the gills with a sharp scissor.

3. Keep the heads in $1 \times$ PBS for 5 min in order to allow bleeding. Note: This drains blood from the tissue, which may perturb further steps in the protocol.

4. Fix the heads overnight at $4{ }^{\circ} \mathrm{C}$ in $4 \%$ paraformaldehyde (PFA) in PBS or $4 \mathrm{hr}$ at room temperature (RT).

5. Wash fixed heads twice with $1 \times$ PBS in a petri dish.

6. Dissect brains carefully in $1 \times$ PBS under a dissecting microscope ${ }^{23}$. The lesion is usually visible under the dissecting microscope. Brains without a visible lesion should be discarded.

7. Transfer brains into $2 \mathrm{ml}$ reaction tubes filled with $1.5 \mathrm{ml} 100 \%$ Methanol $(\mathrm{MeOH})$ and invert the tubes $5 \mathrm{x}$ before incubating them at -20 ${ }^{\circ} \mathrm{C}$ for at least $16 \mathrm{hr}$. Note: The brains can be kept for several months in $\mathrm{MeOH}$ at $-20^{\circ} \mathrm{C}$ until needed for immunohistochemistry or in situ hybridization.

2. Tissue Preparation for Immunohistochemistry

1. Rehydrate the brains through a descending methanol series in $2 \mathrm{ml}$ plastic tubes (sequentially $75 \% \mathrm{MeOH}(1 \times \mathrm{PBS}, 0.1 \% \mathrm{Tween}-20=$ PTW); $50 \% \mathrm{MeOH}, 25 \% \mathrm{MeOH}$. Incubate the brains (15 brains/2 ml reaction tubes) for $5 \mathrm{~min}$ in each solution.

2. Wash the brains in PTW for 5 min. Repeat $4 x$ with fresh PTW.

3. Embedding of the Brains for Sectioning

1. For embedding, transfer the brains from the $2 \mathrm{ml}$ reaction tube in PTW into the cavities of a polyethylene molding cup tray with a transfer pipet (5 mm diameter).

2. Prepare $2 \%$ agarose (DNA grade) in $1 \times$ PBS in an Erlenmeyer flask. Heat it in the microwave at $600 \mathrm{~W}$ until the agarose is dissolved completely. Prepare $50 \mathrm{ml}$ for 10 brains. Let the agarose cool down for $3 \mathrm{~min}$ at room temperature before usage. Keep the agarose on a pre-heated heating block $\left(60^{\circ} \mathrm{C}\right)$ while embedding the brains in order to prevent solidification.

3. Remove the $1 \times$ PBS from the mold containing one brain by using a Pasteur pipette $(1 \mathrm{~mm}$ diameter). Be careful not to damage the brain.

4. Pour agarose into the mold and fill it entirely. Then use a dissection needle to swirl the brain in the agarose to wash away the PTW making it easier to embed the brain.

5. Orient the brain in the mold ventral side down, dorsal side up and place it straight.

6. Let the agarose cool down. For faster cooling, place the mold on ice. Repeat steps. 
7. Repeat 2.3.3-2.3.6 for all brains.

4. Trimming of the Agarose Block

1. Using a dissection needle, pop out the agarose block.

2. With a sharp razor blade, cut the agarose at the posterior end of the brain. Make sure this plane is parallel to the telencephalon.

3. Cut the agarose at the anterior end of the brain. Then flip the block so that it stands on the posterior plane.

4. Remove the excess of agarose by cutting in parallel to the brain's dorsal and ventral sides. Then flip the brain back so that it lies on its ventral side.

5. Cut the block to a truncated triangle in which the telencephalon is located at the smaller plane (Figure 2).

5. Preparing the Vibratome for Sectioning

1. Prepare the vibratome according to the manufacturer's instructions. Use a new razor blade and replace it after 10 brains.

2. Fill the buffer bath with $1 \times$ PBS to a level that just reaches the bottom of the blade.

3. Place a small dot of superglue on the top of the specimen disc of the vibratome.

4. Carefully place the plane that is located posterior to the brain on the superglue. The glue will harden as soon as it is in contact with the $1 \times$ PBS in the vibratome buffer bath.

5. Insert the specimen disc with the specimen into the buffer tray using the manipulator of the microtome.

6. Use the manipulator to rotate the specimen disc into the desired position. Orient the block in the buffer bath in a way that locates the telencephalon just under the surface, with the dorsal part of the brain facing the blade. Then tighten the scrw with a $3 \mathrm{~mm}$ Allen key and remove the manipulator. Note: The clamping screw or one of the clamping devices must not be located over the gap in the specimen disc; in these positions clamping the specimen disc is not possible.

6. Sectioning the Brain

1. Prepare a 24 -well plate to collect the sections. Per brain to be sectioned, fill one well with $1 \mathrm{ml}$ of blocking buffer $(0.1 \%(\mathrm{v} / \mathrm{v}) 10 \%$ Tween-20, $0.2 \%(\mathrm{w} / \mathrm{v})$ bovine serum albumin (BSA), 1\% ( $/ \mathrm{v}$ ) dimethyl sulfoxide (DMSO) in PBS).

2. Start sectioning at $50 \mu \mathrm{m}$ thickness, $1 \mathrm{~mm} / \mathrm{sec}$ speed, and $70 \mathrm{~Hz}$ frequency using a vibrating microtome.

3. Use a synthetic brush $(1 \mathrm{~mm})$ to pick up the thin slices of agarose as they come off the blade and collect them in the 24-well plate.

7. Immunohistochemistry on Vibratome Sections

1. Block non-specific binding sites for $1 \mathrm{hr}$ at RT in blocking buffer $(0.1 \%(\mathrm{v} / \mathrm{v}) 10 \%$ Tween $20,0.2 \%(\mathrm{w} / \mathrm{v}) \mathrm{BSA}, 1 \%(\mathrm{v} / \mathrm{v}) \mathrm{DMSO}$ in PBS).

2. Remove the supernatant and add $250 \mu \mathrm{l}$ of antibody diluted in blocking buffer overnight at $4{ }^{\circ} \mathrm{C}$. Alternatively, incubate the primary antibody for $2 \mathrm{hr}$ at RT. Then wash $3 x$ for $10 \mathrm{~min}$ in PTW. Note: For PCNA staining use a 1:400 dilution (mouse anti-PCNA. For details see table of materials). Multiple primary antibodies can be incubated at the same time.

3. Incubate the sections in secondary antibody for $2 \mathrm{hr}$ at RT then wash $3 \mathrm{x}$ for $10 \mathrm{~min}$ in PTW. Note: 1:1,000 dilution in PTW, for detail see table of materials. Multiple secondary antibodies can be incubated at the same time.

4. Optional: The best results are observed when sections are kept in PTW overnight at $4{ }^{\circ} \mathrm{C}$ in order to allow washing out of nonspecifically bound secondary antibodies. In this case, replace the PTW once again before mounting.

8. Adult Brain RNA in situ Hybridization (alternatively to immunohistochemistry)

1. Brain Dissection and Fixation (same procedure as for immunohistochemistry):

1. Hybridization of probe.

2. Rehydrate brains in $2 \mathrm{ml}$ reaction tubes as described for immunohistochemistry.

3. Incubate brains in proteinase K (ProtK; $10 \mu \mathrm{g} / \mathrm{ml}$ final conc.) in PTW for $30 \mathrm{~min}$. After incubation time remove ProtK/PTW and fix the brains again for $20 \mathrm{~min}$ in BT-Fix (4\% PFA, 4\% sucrose, $\left.0.12 \mathrm{mM} \mathrm{CaCl}_{2}, 77 \mathrm{mM} \mathrm{Na}_{2} \mathrm{HPO}_{4}, 23 \mathrm{mM} \mathrm{NaH}_{2} \mathrm{PO}_{4}\right)$.

4. Wash brains $5 x$ for 5 min in PTW $(0.1 \%(v / v)$ Tween-20 in PBS).

5. Remove PTW and rinse the brains in $500 \mu \mathrm{l} \mathrm{HYB} \mathrm{buffer} \mathrm{(50 \%} \mathrm{(v/v)} \mathrm{deionized} \mathrm{formamide,} \mathrm{5x} \mathrm{SSC,} 500 \mu \mathrm{g} / \mathrm{ml}$ yeast tRNA, $50 \mu \mathrm{g} /$ $\mathrm{ml}$ heparin, $0.1 \%$ Tween-20, $9 \mathrm{mM}$ citricacid). Wait until brains sink to the bottom of the tube then discard the supernatant and add $500 \mu \mathrm{l}$ of clean HYB buffer and incubate at $65-70{ }^{\circ} \mathrm{C}$ for $3-4 \mathrm{hr}$.

6. Dilute the RNA probe to 1:200 or 1:400 (depending on the probe) in $400 \mu$ final volume of HYB buffer. Heat the diluted RNA probe at $70^{\circ} \mathrm{C}$ for $5 \mathrm{~min}$.

7. Remove supernatant from the brains and add the RNA probe, incubate at $65-70^{\circ} \mathrm{C}(\mathrm{O} / \mathrm{N})$. All wash buffer solutions should be prewarmed and kept at $70^{\circ} \mathrm{C}$.

2. Labeling

1. Wash the brains at $65-70{ }^{\circ} \mathrm{C}$ (during all 4 washing steps) with $500 \mu \mathrm{l}$ of wash buffer $1(50 \%$ formamide, $1 \times$ SSC, $0.05 \%$ Tween 20) twice for $30 \mathrm{~min}$. Replace buffer 1 by buffer 2 (2x SSC, $0.1 \%$ Tween 20), and incubate for $15 \mathrm{~min}$. Remove buffer 2 and wash brains in buffer $3(0.2 x$ SSC, $0.1 \%$ Tween 20$)$ for $30 \mathrm{~min}$, repeat this step 2 twice. Replace buffer 3 by buffer $4(0.1 \times$ SSC, $0.05 \%$ Tween 20 in PTW), incubate for 5 min.

2. Remove supernatant and wash for 5 min in $500 \mu$ of PTW at RT.

3. Embed brains in $2 \%$ agarose (in $1 \times$ PBS) and cut vibratome sections $(50-100 \mu \mathrm{m})$ as described for immunohistochemistry.

4. Wash $1 x$ for $5 \mathrm{~min}$ in $500 \mu$ l blocking buffer (1x PBS, $0.1 \%$ Tween-20, $0.2 \%$ (w/v) BSA, 1\% (v/v) DMSO).

5. Remove supernatant and add $500 \mu$ l blocking buffer for $2 \mathrm{hr}$ at RT.

6. Remove supernatant and add $300 \mu \mathrm{l}$ anti-Dig AP-coupled antibody diluted 1:4,000 in blocking buffer.

7. Incubate o/n at $4^{\circ} \mathrm{C}$

3. NBT/BCIP Staining

1. Wash $5 x$ for 15 min with PTW buffer at RT.

2. Rinse $1 \mathrm{x}$ in staining buffer. Wash twice $5 \mathrm{~min}$ in staining buffer $(100 \mathrm{mM}$ Tris/ $\mathrm{HCl} \mathrm{pH} 9.5,50 \mathrm{mM} \mathrm{MgCl}, 100 \mathrm{mM} \mathrm{NaCl}, 0.1 \%$ Tween-20).

3. Remove supernatant and stain with $500 \mu \mathrm{l}$ staining solution ( $3.5 \mu \mathrm{l}$ of 4-Nitroblue tetrazolium chloride (NBT) and 5-Bromo-4chloro-3-indolyl-phosphate (BCIP) per $1 \mathrm{ml}$ staining buffer). 
4. Stop staining by rinsing $3 x$ to $5 x$ with PTW.

9. Mounting of Sections

1. Mount the sections on slides using a water-soluble, non-fluorescent mounting medium (see the Materials table).

2. Keep slides in the fridge at $4{ }^{\circ} \mathrm{C}$. Note: The staining is stable for several weeks or even months.

3. Analyze the slides under a compound or confocal microscope.

\section{Representative Results}

Using the workflow described in this article, a lesion was introduced into the right hemisphere of an adult zebrafish telencephalon

(Figures 1A-1B). A correct wounding leads to a lesion canal that extends from dorsal to ventral through the whole pallium of the telencephalon (Figure 3C). The lesion should be restricted to one of the hemispheres and should not cross the medial ventricle. The lesion canal is visible with bright field microscopy (Figure $3 \mathbf{C}$ ) and will be healed after approximately 35 days ${ }^{15}$.

It was previously shown that after wounding an up-regulation of proliferating cell nuclear antigen (PCNA; Figure 3A) and S100ß (Figure 3B) at 3 to $7 \mathrm{dpl}$ is detectable by immunohistochemistry ${ }^{15}$. The staining of PCNA and S100 $\beta$ is overlapping, indicating proliferation of radial glial cells.

Furthermore, in order to visualize oligodendrocyte precursor cells (OPCs), a stab wound assay was performed on the $T g$ (olig2:EGFP) $\mathrm{fish}^{24}$. Upon staining with an anti-GFP antibody, accumulation of OPCs in close proximity to the lesion canal is detectable (Figure 3D). This accumulation is transient, and OPC clusters are not observed anymore at $35 \mathrm{dpl}^{15}$.

If the lesion was not introduced properly, the lesion canal will not be visible, and up-regulation of PCNA and S100 $\beta$ or accumulation of OPCs will not be detectable. PCNA is the most sensitive marker for brain injury. In order to verify the efficiency of the stab wound and to exclude that both hemispheres are wounded, it is strongly recommended to always stain the sections with an anti-PCNA antibody. In a correctly introduced lesion, PCNA should be significantly up-regulated (up to 4 -fold ${ }^{15}$ ) in only one of the hemispheres. Although in most cases it is sufficient to only use the contralateral unlesioned side as an internal control to monitor changes in gene expression, it is highly recommended to compare the gene expression in the control hemisphere with the expression in telencephalic hemispheres of wild type brains. In this way, any systemic effects of the lesion on gene expression that would affect both hemispheres can be detected.

The stab wound assay in combination with a systematic expression screen of transcription regulators (TRs) $\left(^{25}\right.$ and Schmidt et al., unpublished) can also be used to identify TR genes with a possible role in neurogenesis and regeneration (Figures 4A-4C).

This approach has identified a number of factors that are expressed in the telencephalon and whose expression is specifically up-regulated in response to injury (Figure $\mathbf{4 A - C}$ ).

A

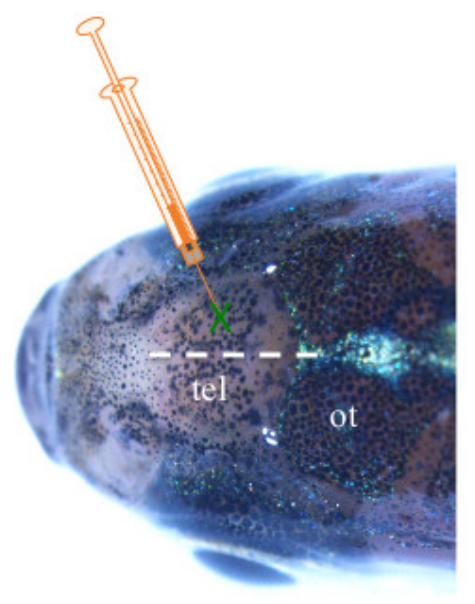

$\mathrm{B}$

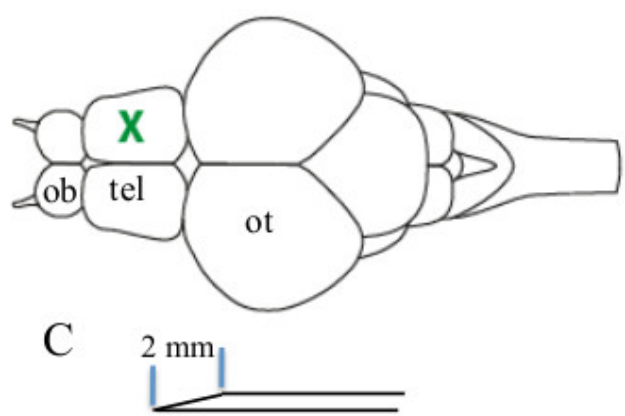

Figure 1. Schematic representation of adult zebrafish telencephalic stab wounding. A) An insulin syringe needle is pushed through the skull of an adult zebrafish to generate a telencephalic injury. The white dashed line represents the border between the two telencephalic hemispheres and the green cross indicates the position of the stab wound. B) Dorsal view of an adult zebrafish brain. The major brain areas are indicated. The green cross shows the position of the induced injury. C) The tip of the needle, which serves to induce the telencephalic injury, measures $2 \mathrm{~mm}$. Olfactory bulb (ob), telencephalon (tel) and optic tectum (ot). 


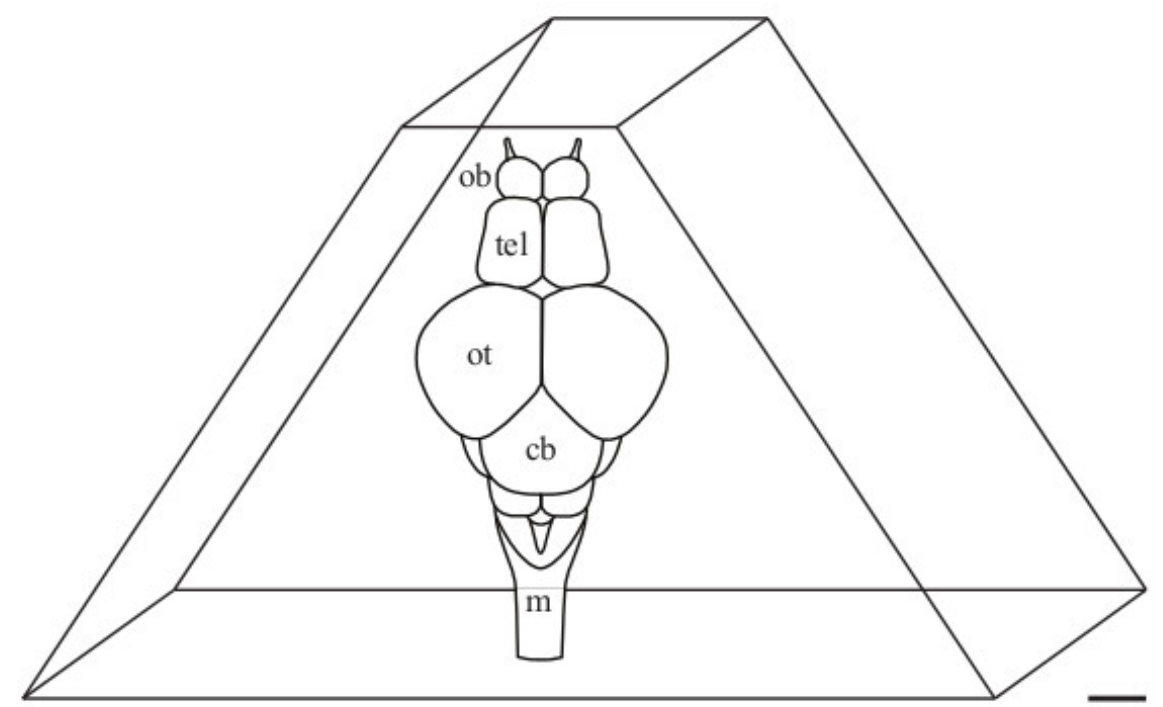

Figure 2. Schematic representation of an adult zebrafish brain in a trimmed agarose block just before vibratome sectioning. Anterior is up. Olfactory bulb (ob), telencephalon (tel), optic tectum (ot), cerebellum (cb) and medulla (m). Scale bar: $500 \mu \mathrm{m}$.
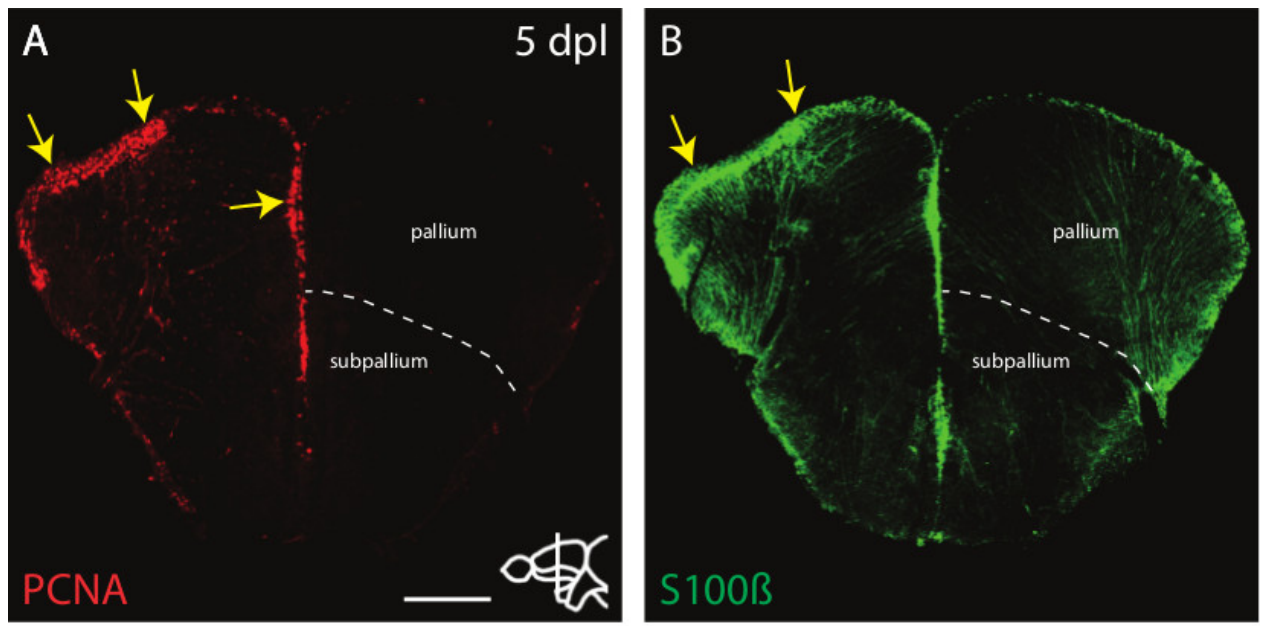

C

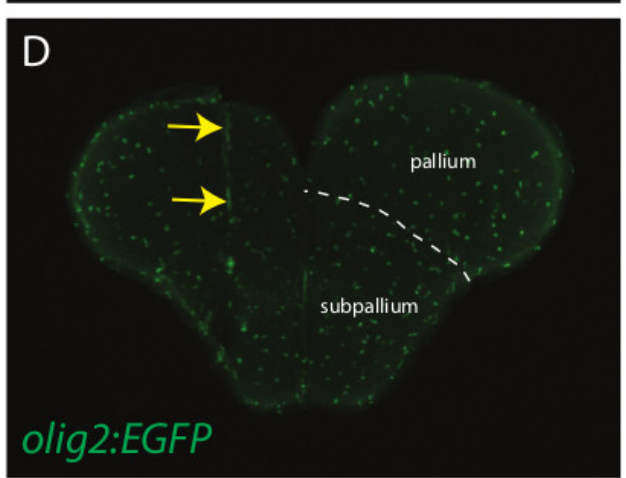

\section{Brightfield}

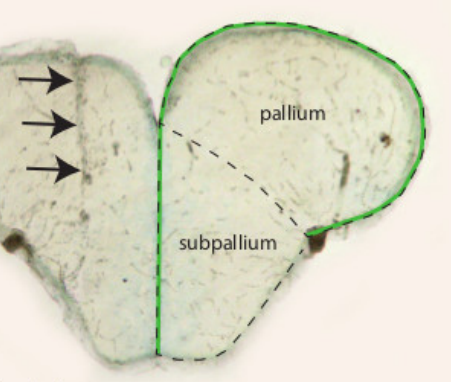

Figure 3. Regenerative responses to stab wound injury. A-D) Adult zebrafish brain at 5 days post lesion (dpl). A-B) The proliferative marker PCNA (A) and the radial glial marker $\mathrm{S} 100 \beta$ (B) are up-regulated at the ventricular zone (arrows) upon stab wounding. (C) The lesion channel is visible under bright field illumination (arrows). The ventricular zone is indicated by the green line. (D) Oligodendrocyte precursor cells (olig2:EGFP ${ }^{+}$) accumulate at the site of lesion (arrows). Scale bar: $200 \mu \mathrm{m}$. In all panels, the right hemisphere is the lesioned hemisphere. Dorsal is up. The dashed line in $A$ to $D$ indicates the border between the pallium and the subpallium. 
A

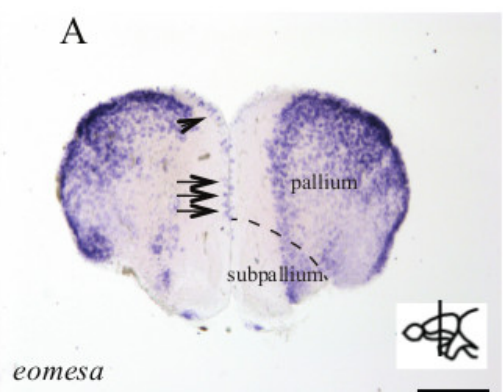

$\mathrm{B}$

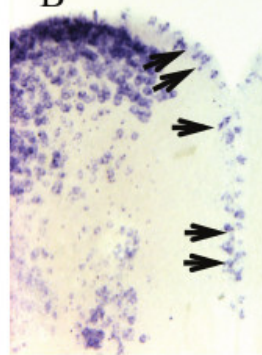

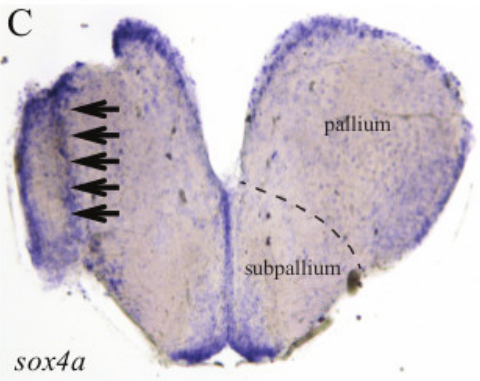

Figure 4. An example of genes up-regulated in response to stab injury. Note the strong up-regulation (arrows) of eomesa (A, B) and sox4a (C) mRNA expression in the right telencephalic hemisphere (stabbed hemisphere) as monitored by in situ hybridization at $5 \mathrm{dpl}$. The left hemisphere is uninjured and serves as control. (B) Shows a higher magnification of the dorsal telencephalic region of A. Dorsal is up. Scale bar: $200 \mu \mathrm{m}$ for $\mathrm{A}$ and $\mathrm{C}$ and $55 \mu \mathrm{m}$ for $\mathrm{B}$. The dashed line in $\mathrm{A}$ and $\mathrm{C}$ indicates the separation between the pallium and the subpallium. Please click here to view a larger version of this figure.

\begin{tabular}{|c|c|}
\hline \multicolumn{2}{|c|}{ ABBREVIATIONS } \\
\hline AP & Alkaline Phosphatase \\
\hline $\mathrm{BCIP}$ & 5-bromo-4-chloro-3'-indolyphosphate \\
\hline BSA & Bovine serum albumin \\
\hline CNS & Central nervous system \\
\hline Dig & Digoxygenin \\
\hline DMSO & Dimethyl sulfoxide \\
\hline dpl & Days post lesion \\
\hline eomesa & Eomesodermin homolog a \\
\hline GFAP & Glial fibrillary acidic protein \\
\hline $\mathrm{hr}$ & Hour \\
\hline HYB buffer & Hybridization buffer \\
\hline $\mathrm{ISH}$ & in situ hybridization \\
\hline $\mathrm{MeOH}$ & Methanol \\
\hline NBT & Nitro-blue tetrazolium \\
\hline OPCs & Oligodendrocyte precursor cells \\
\hline PBS & Phosphate buffered saline \\
\hline PCNA & Proliferating cell nuclear antigen \\
\hline PFA & Paraformaldehyde \\
\hline ProtK & Proteinase $\mathrm{K}$ \\
\hline PSA-NCAM & Polysialylated neuronal cell adhesion molecule \\
\hline PTW & PBS, $0.1 \%$ Tween-20 \\
\hline RNA & Ribonucleic acid \\
\hline RT & Room temperature \\
\hline SGZ & Subgranular zone \\
\hline sox4a & SRY-box containing gene $4 a$ \\
\hline SSC & Saline-sodium citrate \\
\hline SVZ & Subventricular zone \\
\hline TRs & Transcription regulators \\
\hline
\end{tabular}

\section{Discussion}

We describe here a method to induce a telencephalic injury by pushing a needle through the skull of the adult zebrafish, and to analyze the cellular and molecular reaction to this injury by immunohistochemistry and in situ hybridization. The main advantage of this technique over other 
existing approaches is its simplicity, speed and cost efficiency. Therefore, this technique, which allows production of many injured brains in a short time, can be easily combined with a large scale in situ hybridization screen to identify genes with a role in regeneration and neurogenesis.

The age of the adult zebrafish on which the injury is performed is crucial. If the fish are older than 1.5 years, the skull is too hard and it is possible that the skull is being pushed down or fractured rather than cleanly perforated. In contrast, in younger fish (younger than 4 months), there is still a lot of neurogenesis going on, which might lead to false positive results regarding reactive neurogenesis.

Another critical step in this approach is to verify the efficiency of the stab wound and to exclude that both hemispheres are wounded. This can be monitored by staining the sections with the anti-PCNA antibody. In a correctly introduced lesion, PCNA should be significantly up-regulated in only one of the hemispheres.

It is important to bear in mind that the induced mechanical injury will have several side effects such as non-specific cell ablation, increased cell death because of secondary degeneration, inflammation and destruction of the blood-brain barrier, and damage of ventricular zone and as a possible consequence flow of the cerebrospinal fluid into the brain parenchyma. However, the injured fish appear to suffer very little from the injury. Within 2 min they are free swimming again and normal feeding behavior is restored within about 4 hr. After 3 weeks the injury is morphologically not visible anymore. In our hands, from several hundred surgeries we did not lose a single fish despite the fact that we had a $100 \%$ injury rate as assessed by histology at 5 days post-lesion.

In the last years other techniques such as transgenic approaches have been developed in zebrafish to produce tissue or cell type specific ablation $^{2}$. Although these techniques are very elegant and minimally invasive they are based on the generation of complicated DNA constructs and the establishment of several stable zebrafish transgenic lines which can be very tedious and time consuming. Therefore, simple mechanical approaches such as the assay described in this protocol remain an important tool to study adult brain neurogenesis and regeneration.

\section{Disclosures}

The authors have nothing to disclose.

\section{Acknowledgements}

We thank N. Borel and her fish house team, M. Rastegar for help with microscopy, and T. Dickmeis for comments on the manuscript. We are grateful for support by the EU IP ZF-Health, FP7-HEALTH-2007-B2, NeuroXsys, the Interreg Network for Synthetic Biology in the Upper Rhine valley (NSB-Upper Rhine) and the BMBF funded network EraSysBio.

\section{References}

1. Ming, G.L., \& Song, H. Adult neurogenesis in the mammalian brain: significant answers and significant questions. Neuron. 70, 687-702, (2011).

2. Kizil, C., Kaslin, J., Kroehne, V., \& Brand, M. Adult neurogenesis and brain regeneration in zebrafish. Dev Neurobiol. 72, 429-461, (2012).

3. Robel, S., Berninger, B., \& Gotz, M. The stem cell potential of glia: lessons from reactive gliosis. Nat Rev Neurosci. 12, 88-104, (2011).

4. Sofroniew, M.V. Molecular dissection of reactive astrogliosis and glial scar formation. Trends Neurosci. 32, 638-647, (2009).

5. Schmidt, R., Strahle, U., \& Scholpp, S. Neurogenesis in zebrafish - from embryo to adult. Neural Dev. 8, 3, (2013).

6. Zupanc, G.K., \& Sirbulescu, R.F. Adult neurogenesis and neuronal regeneration in the central nervous system of teleost fish. Eur $J$ Neurosci. 34, 917-929, (2011).

7. Zupanc, G.K., \& Sirbulescu, R.F. Teleost fish as a model system to study successful regeneration of the central nervous system. Curr Top Microbiol Immunol. 367, 193-233, (2013).

8. Adolf, B., et al. Conserved and acquired features of adult neurogenesis in the zebrafish telencephalon. Dev Biol. 295, 278-293, (2006).

9. Grandel, H., Kaslin, J., Ganz, J., Wenzel, I., \& Brand, M. Neural stem cells and neurogenesis in the adult zebrafish brain: origin, proliferation dynamics, migration and cell fate. Dev Biol. 295, 263-277, (2006).

10. Zupanc, G.K., Hinsch, K., \& Gage, F.H., Proliferation, migration, neuronal differentiation, and long-term survival of new cells in the adult zebrafish brain. J Comp Neurol. 488, 290-319, (2005).

11. Chapouton, P., Jagasia, R., \& Bally-Cuif, L. Adult neurogenesis in non-mammalian vertebrates. Bioessays. 29, 745-757, (2007).

12. Hinsch, K., \& Zupanc, G.K. Generation and long-term persistence of new neurons in the adult zebrafish brain: a quantitative analysis. Neuroscience. 146, 679-696, (2007).

13. Lindsey, B.W., Darabie, A., \& Tropepe, V. The cellular composition of neurogenic periventricular zones in the adult zebrafish forebrain. $J$ Comp Neurol. 520, 2275-2316, (2012).

14. Marz, M., et al. Heterogeneity in progenitor cell subtypes in the ventricular zone of the zebrafish adult telencephalon. Glia. 58, 870-888, (2010).

15. Marz, M., Schmidt, R., Rastegar, S., \& Strahle, U. Regenerative response following stab injury in the adult zebrafish telencephalon. Dev Dyn. 240, 2221-2231, (2011).

16. Grandel, H., \& Brand, M. Comparative aspects of adult neural stem cell activity in vertebrates. Dev Genes Evol. 223, 131-147, (2013).

17. Ayari, B., El Hachimi, K.H., Yanicostas, C., Landoulsi, A., \& Soussi-Yanicostas, N. Prokineticin 2 expression is associated with neural repair of injured adult zebrafish telencephalon. J Neurotrauma. 27, 959-972, (2010).

18. Baumgart, E.V., Barbosa, J.S., Bally-Cuif, L., Gotz, M., \& Ninkovic, J. Stab wound injury of the zebrafish telencephalon: a model for comparative analysis of reactive gliosis. Glia. 60, 343-357, (2011).

19. Kishimoto, N., Shimizu, K., \& Sawamoto, K. Neuronal regeneration in a zebrafish model of adult brain injury. Dis Model Mech. 5, 200-209, (2012).

20. Kizil, C., et al. Regenerative neurogenesis from neural progenitor cells requires injury-induced expression of Gata3. Dev Cell. 23, 1230-1237, (2012). 
21. Kroehne, V., Freudenreich, D., Hans, S., Kaslin, J., \& Brand, M. Regeneration of the adult zebrafish brain from neurogenic radial glia-type progenitors. Development. 138, 4831-4841, (2011).

22. Kyritsis, N., et al. Acute inflammation initiates the regenerative response in the adult zebrafish brain. Science. 338, 1353-1356, (2012).

23. Gupta, T., \& Mullins, M.C. Dissection of organs from the adult zebrafish. J Vis Exp. (37), (2010).

24. Shin, J., Park, H.C., Topczewska, J.M., Mawdsley, D.J., \& Appel, B. Neural cell fate analysis in zebrafish using olig2 BAC transgenics. Methods Cell Sci. 25, 7-14, (2003).

25. Armant, O., et al. Genome-wide, whole mount in situ analysis of transcriptional regulators in zebrafish embryos. Dev Biol. 380, 351-362, (2013). 